\title{
X-RAY OBSERVATIONS OF ELLIPTICAL GALAXIES BY ASCA
}

\section{SATELLITE}

\author{
K. MATSUSHITA AND K. MAKISHIMA \\ Department of Physics, University of Tokyo \\ 7-3-1,Hongo,Bunkyo-ku,Tokyo,Japan
}

Using ASCA, we have confirmed that the ISM of X-ray bright elliptical galaxies are surprisingly metal poor, as compared to the theoretical predictions. In fact the exact values of the derived metallicity depend considerably on the plasma emission codes. However , the overall metallicity cannot be larger than $\sim 1$ solar. For low $L_{X} / L_{B}$ galaxies, all the available plasma codes suggest abundances less than half a solar. The ASCA spectra may be compatible with somewhat higher metallicity if we assume there is an additional low-temperature component (e.g. $k T e \sim 0.3 \mathrm{keV}$ ). However, the derived abundance can not be over 1 solar. In particular, the Si abundance turns out to be $<1.5$ solar, confirming the metal-poor nature of the ISM. These ASCA results are in severe contradiction with most of the SN Ia rate, particularly that of Tammann (1982). Considering further that a fairly long time $\left(10^{9-10} \mathrm{yr}\right)$ is needed for the stellar mass loss to accumulates into the ISM, it is suggested that the SN Ia rate has remained quite low throughout Hubble time.

Are there plausible mechanisms that reduce the apparent metallicity? Hiding heavy elements in dust is one possibility. However the dust sputtering time is rather short. Dilution of the ISM by the ICM would do the job, but it is inconsistent with the ASCA data since relatively isolated ellipticals also exhibit low abundances. Finally, presence of an unknown continuum source would produce a similar effect, but such a component is not seen in the lowest $L_{X} / L_{B}$ galaxies (Matsushita et al. 1994).

In conclusion, the ISM abundance remains a big puzzle that must be challenged by future investigations, from both theory and observation.

\section{References}

Matsushita, et al. (1994), ApJL, 436, L41 